\title{
A GENERALIZED POINCARÉ STABILITY CRITERION
}

\author{
CARMEN CHICONE AND R. C. SWANSON
}

\begin{abstract}
Let $\Phi_{\ell}^{*} \eta=\Phi^{-t} \circ \eta \circ \phi^{\prime}$ define a semigroup on the Banach space $\Gamma(M, E)$ of continuous sections of $E$ over $M$. It is known that $\left(\Phi^{\prime}, \phi^{\prime}\right)$ is hyperbolic iff $\Phi_{\#}^{t}$ has spectrum off the unit circle for $t \neq 0$. We prove that a third equivalent condition is that the (unbounded!) infinitesimal generator $L$ of $\left\{\Phi_{t}^{*}\right\}$ have its spectrum disjoint from the imaginary axis. In two dimensions this property coincides with the Poincare stability criterion for a periodic orbit of a planar dynamical system.
\end{abstract}

1. Introduction. One may always associate, in a natural fashion, a linear semigroup with a given dynamical system. Thus, if a smooth vector bundle flow $\left(\Phi^{t}, \phi^{t}\right)$ acts on a smooth Riemannian vector bundle $(E, M, \pi)$, we can define the oneparameter group $\Phi_{t}^{\#}$ of bounded operators on the complex Banach space $\Gamma(E)$ of continuous sections $\eta$ of $E$ :

$$
\Phi_{t}^{\#} \eta=\Phi^{-\imath} \circ \eta \circ \phi^{t} .
$$

Certain algebraic properties of this semigroup match up with corresponding qualitative features of the underlying vector bundle flow. The most important such feature is hyperbolicity. Recall that the pair $\left(\Phi^{t}, \phi^{t}\right)$ is hyperbolic if $E$ splits as a continuous Whitney sum of invariant subbundles $E=E^{+} \oplus E^{-}$such that $\Phi^{t}$ contracts $E^{+}$and expands $E^{-}$. Equivalently [4], [6], $\left(\Phi^{t}, \phi^{t}\right)$ is hyperbolic if $\Phi_{t}^{\#}$ for any $t \neq 0$ is a hyperbolic operator; i.e., has spectrum, $\sigma\left(\Phi_{t}^{\#}\right)$, disjoint from the unit circle. It is the goal of this paper to establish that such algebraic or spectral properties have their optimal expression as attributes of the infinitesimal generator of the induced semigroup. The generator of $\Phi_{t}^{\#}$ is a closable operator $L$, densely defined in $\Gamma(E)$ by setting

$$
L \eta=\left.\frac{d}{d t} \Phi_{t}^{\#} \eta\right|_{t=0}
$$

We call an operator infinitesimally hyperbolic if its spectrum is disjoint from the imaginary axis. The principal result of this article is

THEOREM A. $\left(\Phi^{t}, \phi^{t}\right)$ is hyperbolic if and only if $L$ is infinitesimally hyperbolic.

In [1], we established a special case of Theorem $A$ when $\left(\Phi^{t}, \phi^{t}\right)$ is given by the tangent flow $\left(T f^{t}, f^{t}\right)$ of a nonsingular smooth flow $f^{t}$ on $M$ and the vector bundle $E$ is the quotient $T M / X$ with $X=d f^{t} / d t$. Theorem A translates to the statement:

Received by the editors November 15, 1979 and, in revised form, February 28, 1980.

1980 Mathematics Subject Classification. Primary 58F15; Secondary 58F19.

Key words and phrases. Hyperbolic flow, spectrum, adjoint representation. 
the flow $f^{t}$ is Anosov if and only if the Lie derivative $L_{X}$ is infinitesimally hyperbolic on $\Gamma(E)$.

For the tangent flow, if $L_{X}$ is infinitesimally hyperbolic the periodic orbits are isolated; thus, the nonperiodic points are dense. This fact was decisive in our proof of the special case of Theorem A. However, a vector bundle flow can be hyperbolic when $\phi^{t}$ has open sets of periodic points (Proposition 3.1). Because of this fact Theorem A requires a different proof in the vector bundle case which we present in $\S 2$.

Aside from its virtue of generality, the vector bundle formulation of Theorem A offers a true generalization of Poincaré's hyperbolicity criterion for a periodic orbit of a flow on the plane. Recall that if $X$ is a vector field on $\mathbf{R}^{2}$ and $\theta$ is a periodic orbit of the flow of $X$ with period $T$, then $\theta$ is hyperbolic exactly when

$$
\frac{1}{T} \int_{\theta} \operatorname{div}(X) \neq 0 \text {. }
$$

Now, the flow $\phi^{t}$ of $X$ defines a "Poincaré map" $\rho$ from the orthogonal trajectory $\Sigma_{p}$ at $p$ on $\theta$ to any other orthogonal trajectory $\Sigma_{q}$ at $q=\phi^{t}(p)$. If $x$ is a local coordinate for $\Sigma_{p}$ and $y$ is a local coordinate for $\Sigma_{q}$ with $x(p)=0$ and $y(q)=0$, then

$$
\left.\frac{d \rho}{d x}\right|_{x=0}=\exp \left(\int_{0}^{t} \operatorname{div}(X)\left(\phi_{s}(p)\right) d s\right)
$$

In our language this means that the tangent flow on the normal bundle over $\theta$ is representable as the vector bundle flow $F^{t}$ on $S^{1} \times \mathbf{R}$ given by

$$
F^{t}(\theta, v(\theta))=\left(\theta+t, \exp \left(\int_{\theta}^{\theta+t} \operatorname{div}(X) d s\right) v(\theta)\right)
$$

The infinitesimal generator $L$ of $F_{t}^{\#}$ is

$$
L=\frac{d}{d \theta}-\operatorname{div}(X)(\theta)
$$

To compute the spectrum of $L$, assume

$$
\eta^{\prime}-\operatorname{div}(X) \eta=\lambda \eta
$$

for $\eta: S^{1} \rightarrow \mathbf{R}$. Clearly,

$$
\begin{aligned}
\lambda & =\frac{1}{T} \int_{0}^{T} \eta^{\prime} / \eta d \theta-\frac{1}{T} \int_{0}^{T} \operatorname{div}(X) d \theta \\
& =\frac{2 \pi N i}{T}-\frac{1}{T} \int_{0}^{T} \operatorname{div}(X) d \theta
\end{aligned}
$$

where $N \in Z$. Since $\sigma(L)$ consists entirely of eigenvalues ( $L$ is elliptic) $L$ is infinitesimally hyperbolic exactly when

$$
\frac{1}{T} \int_{0}^{T} \operatorname{div}(X) d \theta \neq 0
$$

Hence, Theorem A yields Poincaré's criterion.

In both cases, hyperbolicity is implied by a condition, infinitesimal hyperbolicity, which does not require the integration of the vector field $X$ (cf. $[4$, p. 8]). Of course, 
this expresses the value of Poincaré's criterion and is one of the main motivations for proving Theorem A.

We wish to thank our colleague Nigel Kalton for several very helpful conversations.

2. Proof of Theorem A. The exponential image of the spectrum of the generator $L$ is contained in the spectrum of the operator $\Phi^{\#}=\Phi_{1}^{\#}[3$, p. 467]. Hence, a hyperbolic vector bundle flow induces a group of operators with an infinitesimally hyperbolic generator. To prove Theorem A we must show that an infinitesimally hyperbolic generator induces a hyperbolic group.

Let $x \in M$ and let $E_{x}$ denote the fiber of $E$ at $x$. Theorem A follows from the following proposition.

Proposition 2.1. If $L$ is infinitesimally hyperbolic and $v$ is a nonzero element of $E_{x}$ then

$$
\sup \left\{\left|\Phi_{x}^{t} v\right| \mid t \in \mathbf{R}\right\}=\infty .
$$

In fact, from the general theory of semigroups [3, p. 467], the point spectrum and the residual spectrum of $L$ exponentiate to give the point and residual spectra of $\Phi^{\#}$. Hence, if $\Phi^{\#}$ fails to be hyperbolic there is a complex number $\lambda=e^{i \theta}$ on the unit circle which belongs to the approximate point spectrum $\sigma_{\text {ap }}\left(\Phi^{\#}\right)$ of $\Phi^{\#}$; i.e., a sequence of continuous sections $\eta_{k}$ exists such that for each $k$

$$
\left\|\eta_{k}\right\|=1
$$

and as $k \rightarrow \infty$,

$$
\lim \left\|\Phi^{\#} \eta_{k}-\lambda \eta_{k}\right\|=0
$$

Choose $x_{k} \in M$ such that

$$
\left|\eta_{k}\left(x_{k}\right)\right|=1
$$

If $t \neq 0$ we have

$$
\left|\Phi^{t} \eta_{k}\left(x_{k}\right)-\lambda^{t} \eta_{k}\left(\phi^{t}\left(x_{k}\right)\right)\right|<\left\|\Phi_{t}^{\#} \eta_{k}-\lambda^{t} \eta_{k}\right\|
$$

Hence, as $k \rightarrow \infty$

$$
\lim \left|\Phi^{t} \eta_{k}\left(x_{k}\right)-\lambda^{t} \eta_{k}\left(\phi^{t}\left(x_{k}\right)\right)\right|=0
$$

Now, choose a vector $v$ which is a limit point of the set $\left\{\eta_{k}\left(x_{k}\right)\right\}$ and observe that, choosing a subsequence if necessary, as $k \rightarrow \infty$

$$
\lim \Phi^{t} \eta_{k}\left(x_{k}\right)=\Phi_{x}^{t} v
$$

Therefore,

$$
\left|\Phi_{x}^{t} v\right|<\left|\Phi_{x}^{t} v-\Phi^{t} \eta_{k}\left(x_{k}\right)\right|+\left|\Phi^{t} \eta_{k}\left(x_{k}\right)-\lambda^{t} \eta_{k}\left(x_{k}\right)\right|+\left|\eta_{k}\left(x_{k}\right)\right|
$$

and this implies that $\left|\Phi_{x}^{t} v\right|<2$. Since $t \neq 0$ was arbitrary, we have

$$
\sup \left\{\left|\Phi_{x}^{t} v\right| \mid t \in \mathbf{R}\right\}<\infty
$$

contrary to Proposition 2.1.

For the proof of Proposition 2.1 let BP "denote the set of $\phi^{\prime}$ periodic points $x \in M$ for which there exists an open set $U$ of periodic points and a number 
$K>0$ such that $\theta_{x} \subset U$ and each $y \in U$ has prime period less than $k$. We show first that Proposition 2.1 holds when $v \neq 0$ is in $E_{x}$ and $x \in B P$.

Assume that

$$
\sup \left\{\left|\Phi_{x}^{t} v\right| \mid t \in \mathbf{R}\right\}<\infty
$$

and that the prime period of $x$ is $T>0$. Clearly, $\Phi_{x}^{-T}: E_{x} \rightarrow E_{x}$ is not hyperbolic. Hence, there is a vector $v \in E_{x} \oplus i E_{x}$ and $\theta \in R$ such that

$$
\Phi_{x}^{-T} v=e^{-i \theta T} v
$$

Define, for $0<t \leqslant T$,

$$
\xi\left(\phi^{-t}(x)\right)=e^{i \theta t} \Phi_{x}^{-t} v
$$

Then, $\xi$ is a smooth section on $\theta_{x}$ and a computation shows

$$
\Phi_{T}^{\#} \xi=e^{i \theta T} \xi \text {. }
$$

Again, from the general theory of semigroups [3, p. 467], since $e^{i \theta T}$ is in the point spectrum of $\Phi_{T}^{\#}$, the infinitesimal generator $l$ of $\Phi_{t}^{\#}$ on $\Gamma\left(\Theta_{x}\right)$ has an imaginary number $i \alpha$ in its point spectrum. In particular, there is a smooth section $\xi_{0}$ on $\theta_{x}$ such that $\left\|\xi_{0}\right\|=1$ and $l \xi_{0}=i \alpha \xi_{0}$.

Choose a smooth $\eta \in \Gamma(E)$ which agrees with $\xi_{0}$ on $\theta_{x}$. As $x \in B P$, there is a neighborhood $U$ of $\theta_{x}$ such that the prime period of any point $y \in U$ is less than some finite number $K$. Let $d$ be a distance function compatible with the topology of $M$, and define, for $y \in U$

$$
\delta(y)=\sup \left\{d\left(\phi^{t}(y), \theta_{x}\right) \mid 0<t<K\right\} .
$$

Since, for fixed $t$,

$$
d\left(\phi^{t}(y), \mathcal{O}_{x}\right) \leqslant d\left(\phi^{t}(y), \phi^{t}\left(y_{k}\right)\right)+d\left(\phi^{t}\left(y_{k}\right), \theta_{x}\right)
$$

it follows that

$$
\left|\delta(y)-\delta\left(y_{k}\right)\right|<\sup \left\{d\left(\phi^{t}(y), \phi^{\prime}\left(y_{k}\right)\right) \mid 0<t<K\right\} .
$$

Hence, since $[0, K]$ is compact, as $k \rightarrow \infty$

$$
\lim \delta\left(y_{k}\right)=\delta(y)
$$

i.e., $\delta$ is continuous in $U$. Choose $\varepsilon>0$. As $L \eta-i \alpha \eta \in \Gamma(E)$ there is a neighborhood $V$ of $\theta_{x}$ such that $V \subset U$ and

$$
\sup \{|\operatorname{L\eta }(x)-i \alpha \eta(x)| \mid x \in V\}<\varepsilon .
$$

Finally, find $c>0$ such that $W=\delta^{-1}([0, c]) \subset V$ and define

$$
f(x)=0, \quad x \notin W,
$$

and

$$
f(x)=1-c^{-1} \delta(x), \quad x \in W .
$$

Then, $f$ is a continuous $\phi^{t}$-invariant function on $M$. Hence, $f \eta$ is in the domain of $L$ and

$$
\|L f \eta-i \alpha f \eta\|<\|f\| \sup \{|L \eta(x)-i \alpha \eta(x)| \mid x \in W\}<\varepsilon .
$$

This implies $i \alpha \in \sigma(L)$, contrary to the assumption that $L$ is infinitesimally hyperbolic. The argument has been presented for periodic orbits with period 
$T>0$. However, if $x$ is a rest point in $B P$ and there is a $v \neq 0$ in $E_{x}$ such that

$$
\sup \left\{\left|\Phi_{x}^{t} v\right| \mid t \in \mathbf{R}\right\}<\infty
$$

a similar argument shows that $L$ is not infinitesimally hyperbolic.

To complete the proof we must show Proposition 2.1 when $v \in E_{x}, v \neq 0$ and $x \in M \backslash B P$. Assume that there is a number $K>0$ such that

$$
\sup \left\{\left|\Phi_{x}^{t} v\right| \mid t \in \mathbf{R}\right\}<K .
$$

Observe that if $\left\|\Phi^{\#}\right\|<1$, then $\Phi^{\#}$ is hyperbolic and Theorem $A$ follows. Hence, we may assume $\left\|\Phi^{\#}\right\|>1$.

Choose $\varepsilon>0$. We adapt an argument of Mãné [5, p. 367] to produce a continuous section $\xi$ such that $\|\xi\|=1$ and $\left\|\left(I-\Phi^{\#}\right) \xi\right\|<\varepsilon$. Pick $N \in Z^{+}$such that

$$
N+1 \geqslant 3 K\left\|\Phi^{\#}\right\| / \varepsilon\|v\| .
$$

Since $x \in M \backslash B P$ we can find a point $q$ near $x$ with three properties:

(a) There is a neighborhood $U$ of $q$ such that $\phi^{j}(U) \cap U=\varnothing$ for $j$ an integer with $|j| \leqslant N$,

(b) there is a vector $w \in E_{q}$ such that $|w|=|v|$ and $\left|\Phi_{q}^{j} w\right|<2 K$ for $|j|<N$ and

(c) there is a smooth section $\eta \in \Gamma(E)$ with support in $U$ such that $\eta(q)=w$,

$$
\left|\Phi^{j} \eta(y)\right|<3 K \text { for all } y \in U \text { and }|j|<N .
$$

Define $\xi \in \Gamma(E)$ by (here we correct an error in [5]):

$$
\begin{gathered}
\xi(y)=0 ; \quad y \notin \bigcup_{|j|<N} \phi^{j}(U), \\
\xi(y)=(1-|j| /(N+1)) \Phi^{j} \eta\left(\phi^{-j}(y)\right) ; \quad y \in \phi^{j}(U),|j|<N .
\end{gathered}
$$

Then

$$
\left\|\left(I-\Phi^{\#}\right) \xi\right\|<\varepsilon\|\xi\| .
$$

Therefore, a sequence $\eta_{k} \in \Gamma(E)$ exists such that

(a) $\left\|\eta_{k}\right\|=1$,

(b) as $k \rightarrow \infty, \lim \left\|\left(\Phi^{\#}-I\right) \eta_{k}\right\|=0$ and

(c) for $N \in Z^{+}$, there is an integer $N_{0}$ such that for each $k>N_{0}$ a point $q \in M$ exists which is nonperiodic or has prime period larger than $N$ with $|\eta(q)|=1$.

Choose $\varepsilon>0$ and pick $N>(8 c+\varepsilon) / \varepsilon$ where

$$
c=\sup \left\{\left\|\Phi_{t}^{\#}\right\| \mid 0<t<1\right\} .
$$

Then, there is a $k$ so large that

(a) $\left\|\Phi^{\#} \eta_{k}-\eta_{k}\right\|<\varepsilon / 4 c$ and

(b) a point $x_{0}$ and a cross-section $\Sigma$ at $x_{0}$ exists such that

$$
\left|\eta_{k}\left(x_{0}\right)\right|=1
$$

and $\Sigma \times[-N, N]$ is a flow box for $\phi^{t}$.

With these choices, the construction in [1, Theorem 2.1] produces a smooth section $\eta \in \Gamma(E)$ such that

$$
\|\eta\|=1
$$


and

$$
\|L \eta\|<\varepsilon
$$

Hence, $0 \in \sigma(L)$ contrary to the assumption. This completes the proof of Proposition 2.1. Q.E.D.

3. Existence of hyperbolic flows. The following proposition shows that hyperbolic vector bundle flows exist over any given flow.

Proposition 3.1. If $(F, M, \rho)$ is a vector bundle with compact base manifold $M$ and $\phi^{t}$ is a smooth flow on $M$, then there is a vector bundle flow $\left(\Psi^{t}, \phi^{t}\right)$ in $(F, M, \rho)$ which is hyperbolic.

Proof. Choose a fiber metric $g$ for $F$ and let $\nabla$ denote the associated metric connection. If $X=d \phi^{t} / d t$ and $I$ denotes the identity on $F$, define

$$
A=\nabla_{X}+I \text {. }
$$

$A$ generates a strongly continuous group $T(t)$ of operators in $\Gamma(F)$. Since $\nabla_{X}$ and $I$ commute, $T(t)$ has the form

$$
T(t)=e^{t I} P_{t}=P_{t} e^{t I}
$$

where for $\eta \in \Gamma(F), P_{t} \eta(x)$ is the vector in $E_{x}$ obtained by parallel transport of the vector $\eta\left(\phi^{t}(x)\right)$ along $\phi^{t}(x)$ to $x$. Clearly, $T(t)$ also has the form $\Psi_{t}^{\#}$ for the vector bundle flow $\left(\Psi^{t}, \phi^{t}\right)$ where

$$
\Psi_{x}^{t}: E_{x} \rightarrow E_{\phi^{\prime}(x)}
$$

is the mapping "parallel transport along $\phi^{t}(x)$ followed by $e^{t I}$." Moreover, in terms of the norm in $\Gamma(F)$ induced by $g, P_{t}$ is an isometry of $\Gamma(F)$. Since $e^{t I}$ is hyperbolic and $e^{t I}$ commutes with $P_{t}, \Phi_{t}^{\#}$ is hyperbolic. Q.E.D.

\section{REFERENCES}

1. C. Chicone and R. C. Swanson, The spectrum of the adjoint representation and the hyperbolicity of dynamical systems, J. Differential Equations 36 (1980), 28-40.

2. D. B. A. Epstein, Periodic flows on three-manifolds, Ann. of Math. 95 (1972), 66-82.

3. E. Hille and R. S. Phillips, Functional analysis and semi-groups, Amer. Math. Soc. Colloq. Publ., Vol. 31, Amer. Math. Soc., Providence, R. I., 1957.

4. M. Hirsch, C. Pugh and M. Shub, Invariant manifolds, Lecture Notes in Math., vol. 583, Springer-Verlag, Berlin and New York, 1977.

5. R. Mañé, Quasi-Anosov diffeomorphisms and hyperbolic manifolds, Trans. Amer. Math. Soc. 229 (1977), 351-370.

6. J. Mather, Characterization of Anosov diffeomorphisms, Indag. Math. 30 (1968), 479-483.

Department of Mathematics, University of Missouri, Columbia, Missouri 65211 